\title{
3 Research Square \\ ROR2 Promotes EMT and Necrosis by \\ Hyperactivating ERK in Melanoma
}

\author{
María Victoria Castro
}

Universidad Maimonides

\section{Gastón Barbero}

Universidad Maimonides

María Belen Villanueva

Universidad Maimonides

Jérémie Nsengimana

Newcastle University

Julia Newton-Bishop

Leeds Institute of Medical Research

\section{Edith Illescas}

Universidad Maimonides

María Josefina Quezada

Universidad Maimonides

Pablo Lopez-Bergami ( $\nabla$ lopezbergami.pablo@maimonides.edu )

Universidad Maimonides https://orcid.org/0000-0001-8669-6931

\section{Research Article}

Keywords: ROR2, ERK, Melanoma, EMT, Migration, Invasion, Tumor necrosis.

Posted Date: November 17th, 2021

DOI: https://doi.org/10.21203/rs.3.rs-1050315/v1

License: (c) (i) This work is licensed under a Creative Commons Attribution 4.0 International License.

Read Full License 


\section{Abstract}

\section{Purpose}

Receptor tyrosine kinase-like orphan receptor 2 (ROR2) has been shown to play opposite roles in the progression of different tumor types. In melanoma, ROR2 was shown to associate with a more invasive phenotype and to contribute to experimental lung colonization. However, the underlying mechanisms regulated by ROR2 have not been elucidated. More importantly, it has not been established whether ROR2 is just a marker or a driver of melanoma aggressiveness.

\section{Methods}

Gain and loss-of-function experiments were applied to study the biological function of ROR2 in melanoma. Transwell assays and Western blots were used to evaluate cell migration and both expression and activation of Epithelial-Mesenchymal Transition (EMT) markers and signaling proteins. The role of ROR2 in vivo was assessed in xenotransplantation experiments.

\section{Results}

We describe that ROR2 promotes EMT by inducing cadherin switch and the upregulation of the transcription factors ZEB1, Twist, Slug, Snail, and HIF1A, together with a mesenchymal phenotype and increased migration. ROR2 association with EMT is also observed in melanoma samples. ROR2 exerts these effects by hyperactivating the MAPK/ERK pathway far above the typical high constitutive activity observed in melanoma. ROR2 also promoted EMT, invasion, and necrosis in xenotransplanted mice. This important role of ROR2 translates into melanoma patient's prognosis since patients with lymph node metastasis displaying elevated ROR2 levels have reduced overall survival and distant metastasis-free survival.

\section{Conclusions}

These results demonstrate that ROR2 contributes to melanoma progression by hyperactivating ERK and inducing EMT and necrosis. Thus, ROR2 can be an attractive therapeutic target for metastatic melanoma.

\section{Introduction}

Melanoma is responsible for $80 \%$ of skin cancer-related deaths. Furthermore, melanoma incidence rates have been increasing steadily for several decades and it currently accounts for $6 \%$ of new cancer cases in the United States [1]. Although the survival of melanoma patients has improved significantly since the implementation of both targeted and immune-based treatments [2], a large fraction of patients are resistant or not eligible for the current therapeutical approaches, highlighting the need to identify novel targets to improve melanoma therapy. A major obstacle toward this goal is our poor understanding of the molecular mechanisms leading to metastasis, the primary cause of death of cancer patients. 
Epithelial-mesenchymal transition (EMT) is a process through which epithelial tumor cells undergo profound morphological, biochemical, and transcriptomic changes to acquire a mesenchymal cell phenotype. EMT is critical in cancer since the resulting mesenchymal-type tumor cell possess enhanced migratory capacity, invasiveness, resistance to apoptosis, and production of extracellular matrix (ECM) components which results in greater metastatic dissemination and therapy resistance [3]. Although EMT was initially described for epithelial tumors, it has been shown that non-epithelial tumors, including melanoma, experience a similar process $[4,5]$. In cancer, EMT is induced by hypoxia, cytokines, and growth factors that promote the activation of several intracellular pathways including the phosphoinositide 3-kinase (PI3K)/Akt, mitogen-activated protein kinase (MAPK), SMAD, or nuclear factorkappa B (NF-KB) [6]. In turn, these pathways activate EMT-transcription factors (EMT-TFs) from the Snail, Twist, and ZEB families, which regulate the expression of genes that establish the mesenchymal phenotype [7].

Receptor tyrosine kinase-like orphan receptor 2 (ROR2) is a receptor for the Wnt5a ligand that plays a major role during embryonic development but is strongly downregulated after birth [8]. ROR2 expression is often dysregulated in human cancers and, similar to Wnt5a, its functions vary depending on the cancer type, functioning either as a tumor-suppressor or as a tumor-promoter [9]. ROR2 played as a tumor promoter in non-small cervical cancer [10], renal cell carcinoma [11, 12], colon cancer [13], melanoma [14], and breast cancer [15]. In contrast, ROR2 has anti-oncogenic functions in endometrial [16], colon [17], and gastric [18] cancer as well as in medulloblastoma [19] and hepatocellular carcinoma [20]. The effect of ROR2 in cancer is mediated by the regulation of cell proliferation, cell migration and invasion, and in vivo tumor growth, which are modulated either positively or negatively depending on the tumor type. In melanoma, ROR2 has been scarcely investigated. It was shown that Ror2 enhanced the migration and metastasis ability of B16 murine melanoma cells in a Src-dependent manner that is negatively regulated by Neurotrophin receptor-interacting MAGE homolog (NRAGE) [21]. In addition, ROR2 expression associates with a more invasive phenotype [14] and is required for the pro-metastatic effect of Wnt5A on melanoma cells [22]. However, it is still not clear weather ROR2 is just a marker or a driver of melanoma invasiveness. In the event that the latter is correct, it is necessary to establish the underlying mechanisms. In the present study, we have evaluated the hypothesis that ROR2 promotes melanoma progression by inducing EMT, a process with wide-ranging consequences for the progression of melanoma [7, 23].

\section{Results}

\section{ROR2 promotes EMT in melanoma cell lines}

To investigate the role of ROR2 in melanoma progression we used both gain- and loss-of-function approaches based on A375 and UACC903 cell lines overexpressing ROR2 (Fig. 1A) and MeWo and M2 cell lines with ROR2 silencing (Fig. 1B). Upon generation of these cell lines, we observed that ROR2 expression induced marked morphological changes in both A375 and MeWo cells (Fig. 1C). This effect was also detected, albeit less noticeably, in UACC903 and M2 cells (data not shown). Compared to control cells, A375-ROR2 cells presented a more elongated spindle morphology, were more scattered, and 
made cell-cell contacts more randomly. Measurement of morphometric parameters revealed an increase in elongation and a decrease in roundness in A375-ROR2 cells (Fig. 1D). On the other hand, the typically thin and elongated MeWo cells turn to a more compact shape upon ROR2 silencing (Fig. 1C) that was evidenced by a decrease in elongation and an increase in roundness (Fig. 1D). The morphological features of ROR2 expressing cells induced by ROR2 are similar to those described in cells undergoing EMT [24]. Although neural crest-derived melanocytes are not epithelial in origin, an EMT-like process is a well-documented phenomenon contributing to the metastatic potential of melanoma [7]. To further evaluate the induction of EMT by ROR2 we determined the expression of both epithelial and mesenchymal markers upon ROR2 overexpression and silencing. We found that ROR2 decreased the expression of the epithelial marker E-cadherin and increased the expression of both the mesenchymal markers N-cadherin and Vimentin and the EMT-inducing transcription factors Twist, Slug, ZEB1, and Snail [25](Fig. 1E). In contrast, the expression levels of these proteins were altered in the opposite direction by ROR2 knocking-down in both M2 and MeWo cells (Fig. 1F). Furthermore, both overexpression and silencing experiments confirmed that ROR2 positively regulates the protein levels of HIF-1a, another major EMT driver (Fig. 1G). Finally, we measured ROR2-dependent changes in cell migration as a cellular read-out of EMT induction. We determined that ROR2 overexpression in both A375 and UACC903 cells increased cell migration (Fig. 1H and Suppl. Fig. S1). In contrast, silencing of ROR2 decreased cell migration in M2 cells (Fig. 1I). These results demonstrate that ROR2 promotes EMT in melanoma cells.

\section{ROR2 promotes EMT in melanoma patients}

In a previous report, we described six prognostic gene expression signatures (LMC classes) from the analysis of a large cohort of primary melanoma patients (LMC, Leeds Melanoma Cohort) [26]. Among the six classes, ROR2 is highest in class 6, characterized by having a high EMT score, upregulation of both cJUN and AXL, and a bad prognosis [26] (Fig. 2A). To confirm this role of ROR2 in EMT, we sought to identify Differentially Expressed Genes (DEGs) between melanoma samples from the independent dataset TCGA-SKCM with high and low ROR2 levels. In agreement with the observations above, the DEGs upregulated in tumors with high ROR2 expression (Supplementary Table S1) were significantly enriched in signatures associated with invasion, extracellular matrix organization, and EMT (Fig. 2B). Altogether, these results demonstrate that ROR2 promotes EMT in human melanoma.

\section{Induction of EMT by ROR2 is mediated by ERK hyperactivation}

Since it was recently shown that p38 signaling promoted EMT in breast cancer downstream of ROR2 [27], we evaluated the effect of ROR2 in p38 phosphorylation in melanoma cells. We found that ROR2 overexpression increased p38 phosphorylation and ROR2 silencing inhibited this pathway (Suppl. Fig. S2). However, inhibition of p38 using the pharmacological inhibitor SB202190 did not alter the expression of the EMT marker Vimentin (Suppl. Fig. S3). These results suggest that although ROR2 activates MAPK/p38, this pathway is not implicated in EMT in melanoma. Next, we looked at the effect of ROR2 on the MAPK/ERK pathway. Despite melanoma cells present constitutive activation of ERK, we found that ROR2 overexpression increased around 10-fold the phosphorylation of ERK (Fig. 3A). In contrast, 
silencing of ROR2 abolished ERK phosphorylation (Fig. 3B). To determine the role of ERK on EMT induction by ROR2 we used PLX-4032 (PLX), an inhibitor of the ERK pathway. Before doing so, we corroborated that $10 \mathrm{mM}$ PLX similarly inhibited p-ERK levels in both A375-Empty and A375-ROR2 (Fig. 3C). The addition of PLX induced the expression of E-cadherin and potently inhibited Vimentin and Ncadherin expression in A375-ROR2 cells but not in A375-Empty cells (Fig. 3D). PLX also inhibited Snail levels in both cell lines although the inhibition observed in A375-ROR2 was much potent (Fig. 3D). Similarly, PLX abrogated both basal and ROR2-induced HIF-1a levels (Fig. 3E). These results demonstrate that ROR2 induces ERK hyperactivation and that the induction of EMT by ROR2 is highly dependent on this event.

\section{ROR2 induces in vivo EMT, invasion, and necrosis}

To evaluate the role of ROR2 in vivo we injected both A375-Empty and A375-ROR2 cells into nude mice. After 6 weeks, tumors from A375-ROR2 were significantly smaller than those from control mice (Fig. 4A). Histological analysis of tumor sections revealed that the tumor cells from mice injected with A375-Empty had a cobblestone-like shape whereas those from the A375-ROR2 derived tumors had an elongated shape (Fig. 4B), suggestive of epithelial-like and mesenchymal-like phenotypes, respectively. In line with this observation, western blot analysis revealed that A375-ROR2 tumors express low levels of the epithelial marker E-cadherin and high levels of the mesenchymal markers Vimentin, N-cadherin, ZEB1, Twist, Slug, Snail, and Fibronectin together with higher expression of p-ERK (Fig. 4C). The upregulation of p-ERK, Vimentin, and Snail in A375-ROR2 tumors was confirmed by immunohistochemistry of tumor sections (Fig. 4D). In agreement with this mesenchymal profile and the increased in vitro migration of A375-ROR2 cells, tumors from mice injected with these cells were found to invade adjacent muscles (Fig. $4 \mathrm{E})$.

Interestingly, unlike tumors from control mice, those from mice injected with A375-ROR2 cells presented extensive necrosis (Fig. 5A). The induction of necrosis was confirmed by the observation of an increased expression of the necrotic markers 14-3-3 and HSP90 [28] (Fig. 5B) and necrotic PARP cleavage [29] (Fig. 5C). The necrotic areas from A375-ROR2 tumors were delimited by cells presenting high levels of both $p$ ERK and Snail nuclear staining (Fig. 5D). In contrast, the cells delimiting the small regions of necrosis present in control tumors displayed both negative Snail and low p-ERK staining (Fig. 5D). These results demonstrate that ROR2 promotes in vivo necrosis, EMT, and tumor cells with invasive features.

\section{ROR2 expression at latter stages reduces melanoma survival}

Following our observation that ROR2 promotes EMT and invasion and previous reports showing that ROR2 increased lung colonization in mouse models of tail vein injection, we sought to evaluate whether ROR2 contributes to metastasis in melanoma patients. To this end, we determined the impact of ROR2 levels on melanoma-specific overall survival, a direct indicator of distant metastasis. To avoid the confounding effect of comparing primary tumors with ample differences in their clinico-pathological features (i.e. AJCC stage) we restricted our analysis to patients with lymph node metastasis (LNM), an early step on the metastatic cascade that is also a good indicator of distant metastasis and survival. 
Thus, the relationship between ROR2 levels in LNM samples and survival was evaluated in a cohort of 130 samples from dataset GSE65904. High levels of ROR2 significantly correlated with shorter overall survival (Fig. 6A). Although ROR2-high and ROR2-low patients presented similar distant metastasis-free survival (dMFS), the subgroup of patients with the highest ROR2 levels (Z-score $>1$ ) exhibited a significantly shorter dMFS (Fig. 6B). These results indicate that tumors expressing high levels of ROR2 are prone to form metastasis and have shorter survival. Furthermore, ROR2 not only favors metastasis but its expression is also important in established metastasis as revealed by transcriptomic data showing that $R O R 2$ levels are significantly higher in metastasis than in primary tumors in both the TCGA-SKCM and GSE46517 datasets (Fig. 6C and 6D). Moreover, analysis of matched primary and metastatic samples from the same patients revealed a significant ROR2 increase in the latter with seven out of nine patients displaying higher ROR2 levels in the metastatic sample (Fig. 6E). Altogether, our results demonstrate that ROR2 promotes EMT and invasion and contributes to melanoma metastasis.

\section{Discussion}

ROR2 plays a dual role in cancer, either by acting as a tumor suppressor or an oncogene depending on the tumor type [9]. ROR2 exerts these roles by regulating either positively or negatively various processes including proliferation, invasion, tumor growth, and EMT. Regarding this last process, the investigations published so far have revealed opposites roles for ROR2. In both invasive ductal carcinoma and esophageal squamous cell carcinoma, ROR2 was shown to inhibit EMT through repressing $\beta$-catenin and Akt signaling [30]. In contrast, ROR2 promoted EMT following activation of p38 in breast cancer [27] and of Rac1 and RhoA in ovarian cancer [31]. Meanwhile, other studies concluded that although ROR2 promoted cell motility and invasiveness, it did not affect the expression of EMT markers in both squamous cell carcinoma [32] and the epidermoid carcinoma cell line A-431 [33].

In melanoma, it was shown that ROR2 silencing in both mouse B16 cells $[14,21]$ and human M93-047 cells [14] reduced lung colonization of cells after intravenous injection, suggesting that ROR2 is important for melanoma metastasis. In addition, ROR2 expression was found to be upregulated in melanoma cells with an invasive phenotype [14], providing a plausible explanation for its role in metastasis. However, the precise cellular and molecular mechanisms regulated by ROR2 have not been studied. In the present manuscript, we demonstrate that ROR2 promotes melanoma progression by inducing EMT and provide a detailed characterization of the underlying mechanism. We have described that ROR2 promotes both morphological changes toward a mesenchymal phenotype and the expression of EMT markers such as Vimentin and N-cadherin as well as of the prototypical EMT transcription factors Snail, ZEB1, Twist, and Slug. In addition, we described that ROR2 promotes in vitro migration and in vivo invasion. We also demonstrated that ROR2 was associated with EMT induction in both primary and metastatic melanoma samples. Among the six classes of stage I melanoma tumors identified by transcriptomic analysis of the LMC, ROR2 expression was highest in class 6, the only one presenting an EMT expression signature. Furthermore, DEGs upregulated in melanoma tumors expressing high ROR2 were enriched in signatures of EMT and migration. 
We have determined that ROR2 promotes EMT by activating the phosphorylation and activity of ERK and the subsequent upregulation of EMT-related proteins. Notably, the relationship between ROR2 and the MAPK/ERK pathway had not been explored in depth to this date. Only a recent article, described that ROR2 is required for Wntless-dependent activation of ERK in neuroendocrine prostate cancer [34]. Despite melanoma cells present high constitutive activity of ERK, overexpression of ROR2 increased p-ERK levels even further. In addition, silencing of ROR2 abrogates p-ERK levels providing evidence of the striking capacity of ROR2 to manipulate the activity of such a critical pathway. The ability of ROR2 to hyperactivate ERK is reminiscent of the increase in p-ERK levels observed in melanoma cells addicted to MAPK inhibitors upon drug withdrawal. Interestingly, in both hyperactive ERK models, excessive ERK activity is associated with an EMT-like switch [7, 35, 36] and a deleterious effect on melanoma cells by inducing cell death via necrosis or parthanatos [35-37]. In our model, we have observed that tumors generated upon injection with A375-ROR2 cells present abundant necrosis. We have ruled out tumor hypoxia as the culprit not only because of the reduced size of the tumors (compared with that of control mice) but primarily because we showed that ROR2 promotes HIF-1a upregulation. Interestingly, the necrotic areas were surrounded by intense nuclear staining of both $p$-ERK and Snail. This observation is consistent with both a Snail positive feedback loop with nuclear p-ERK [38] and the induction of metabolic stress-induced necrosis as demonstrated in other tumor types [39, 40]. Since necrosis is often associated with metastasis and poor prognosis of cancer patients [41] this finding add another mechanisms to the tumor progressing effects of ROR2 in melanoma.

It has been shown that ROR2 is a HIF-1 a target [42]. Along these lines, O'Connell et al showed that melanoma cells with an invasive phenotype induced by a hypoxic stimulus, present upregulation of ROR2 [22]. Our results suggest a far more important role for ROR2. Instead of being a marker of a more invasive phenotype that requires a hypoxic environment to be upregulated, our data demonstrate that ROR2 is a driver of EMT and migration in normoxic conditions. Moreover, our data reveal that ROR2 is a potent inductor of HIF-1a in normoxia. This not only suggests a positive feedback loop between ROR2 and HIF$1 \mathrm{a}$ but also that this loop, driven by ROR2 upregulation, may take place before the tumor becomes hypoxic.

The few previous studies that investigated ROR2 in melanoma agreed that it contributes to melanoma metastasis, following the analysis of lung colonization experiments upon intravenous injection of cells $[14,21]$. Following these observations and our findings on the role of ROR2 in EMT, we wanted to determine the role of ROR2 in human melanoma metastasis. To this end, we focused on the development of systemic metastasis in patients with regional melanoma spread. The reasons why we did not evaluate the spread from primary tumors to distant metastasis are two-fold. First, because as opposed to patients with regional spread (i.e. LNM), most primary tumors will never form distant metastasis. The second reason is that primary tumors have many confounding variables affecting metastasis that can interfere with the assessment of ROR2's role. In contrast, many of these primary tumor features (i.e. tumor thickness) are much less relevant for melanoma prognosis when lymph nodes spread had already occurred. It is important to stress that the use of patients with LNM does not imply introducing a bias with respect to ROR2 expression. Given that lymphatic dissemination does not require EMT, both ROR2- 
high and ROR2-low primary tumors are equally capable of forming LNM. Our analysis revealed that high expression of ROR2 in LNM correlates with shorter overall and distant metastasis survival. This allows us to speculate that, regardless of the debate about whether distant metastasis derives from the primary tumor or LNM, increased ROR2 expression at this stage favors enhanced ERK activity, migration, and EMT, leading to a worse prognosis. It should be noted, however, that we did not find any association between ROR2 and the overall survival of metastatic samples from the TCGA-SKCM dataset (Supplementary Fig. S4). A critical difference between the two cohorts that might explain the different results is that the TCGA dataset is composed largely of samples from distant metastasis and therefore their ROR2 expression values correspond to a time frame where systemic spread had already occurred.

Several pieces of evidence demonstrate that ROR2 expression is significantly higher in metastasis than in primary melanomas. These include data from melanoma tissue microarrays [14], analysis of mRNA expression on large cohorts of patients, and data from primary and metastatic matched samples from the same patients (this article). The high expression of ROR2 in metastatic tissues might indicate that beyond promoting EMT and metastasis, this receptor has additional functions in metastatic outgrowths. This observation, together with the role of ROR2 described here and in previous publications, encourages the use of ROR2 as a therapeutic target for melanoma. ROR receptors were already proposed as potential targets due to their restricted expression in adult tissues and their surface location [8]. Accordingly, several monoclonal antibodies targeting ROR1 are currently in advanced clinical trials (NCT02706392, NCT02776917, NCT03088878, NCT04441099). Likewise, the effectiveness of targeting ROR2 is being evaluated in phase 1 and 2 clinical trials by using ROR2 antibody-drug conjugate (NCT03504488) and Chimeric Antigen Receptor T (CAR-T) cell therapy (NCT03393936 and NCT03960060) in several solid tumor types. Our findings indicates that ROR2 is a therapeutic target in melanoma and warrant further testing in clinical trials.

\section{Conclusions}

We have shown that ROR2 promotes migration, invasion, necrosis and EMT in melanoma. Mechanistically, induction of EMT is strongly dependent on hyperactivation of the ERK pathway and necrosis is associated with both nuclear p-ERK and Snail expression. In addition, we demonstrated that ROR2 expression correlates with EMT in melanoma tumor samples. Further, we show that ROR2 levels increased in metastasis compared with primary tumors and that expression of ROR2 in LNM correlates with both overall survival and dMFS. These findings indicates that ROR2 is a promising therapeutic target in melanoma.

\section{Materials And Methods}

\section{Cell culture.}

Melanoma lines were provided by Dr. Zeev Ronai (Sanford Burnham Prebys Medical Discovery Institute) except for the M2 cell line that was provided by Sergio Alvarez (IMIBIO-CONICET) [43]. Cells were 
maintained in DMEM supplemented with 10\% fetal bovine serum (FBS, Gibco), $100 \mathrm{U} / \mathrm{ml}$ penicillin and $100 \mathrm{mg} / \mathrm{ml}$ streptomycin (Invitrogen), at $37^{\circ} \mathrm{C}$ and $5 \% \mathrm{CO}_{2}$. The cell lines were free of mycoplasma contamination and were authenticated by short tandem repeat analysis as described [44]. The concentrations of PLX4032 (10618, Cayman Chemicals) and SB202190 (10010399, Cayman Chemicals) were $10 \mu \mathrm{M}$ and $15 \mu \mathrm{M}$, respectively. DMSO was used as control.

\section{shRNA constructs, over-expression system and viral infection}

The shRNAs against ROR2 were described previously [45]. The targeting sequences are C2 shRNA 5'CTGGGTGTATGCCCTCATGAT-3' and C4 shRNA 5'-CCCTGGTGCTTTACGCAGAAT-3'. Human ROR2 was cloned into the VIRSP vector. Generation of viral particles and stable transduction were performed as described $[46,47]$.

\section{Western blotting.}

Cell lysates were collected and processed as described [46]. The antibodies used are described in Supplementary Table S2. The band intensities in the phosphoprotein blots were normalized with those of the total proteins obtained from the same blots after strippingand reprobing. To draw a conclusion in a particular experiment at least three biological (independent) replicates of paired samples were examined to calculate the mean and standard deviation. The log transformation of FC values was calculated to obtain a more symmetric distribution that better suits the normality assumptions of the subsequent $\mathrm{t}$-test. Tumor tissue from xenotransplanted mice were minced from the surrounding skin, homogenized and embedded in RIPA Buffer supplemented with protease and phosphatase inhibitors in a relation of $350 \mu$ Buffer $/ 20 \mathrm{mg}$ tissue. The mixture was incubated for 30 minutes in agitation at $4^{\circ} \mathrm{C}$. Finally, it was centrifuged at $10000 \mathrm{rpm}$, at $4^{\circ} \mathrm{C}$ for 10 minutes, and supernatant was recovered and stored at $-20^{\circ} \mathrm{C}$.

\section{In vivo assays.}

$\mathrm{N}: \mathrm{NIH}(\mathrm{S})$-nu nude mice were injected subcutaneously with $2 \times 10^{6} \mathrm{~A} 375$-Empty or -ROR2 cells and tumor growth was monitored twice a week. Tumor sizes were measured using a caliber, and tumor volumes were calculated using the formula: volume $=(2 \mathrm{x}$ width $\mathrm{x}$ length $) / 2$. Tumors were harvested, paraffin embedded and sectioned.

\section{Immunohistochemistry.}

Immunohistochemistry staining of tissue samples was performed as described [48]. Antigen retrieval was by heat-induced epitope retrieval using Citrate Buffer $(10 \mathrm{mM}, \mathrm{pH} 6)$ for ROR2, Vimentin, and Snail or TrisEDTA Buffer (Tris $10 \mathrm{mM}$ and EDTA $1 \mathrm{mM}, \mathrm{pH}$ ) for $\mathrm{p}$-ERK at $100^{\circ} \mathrm{C}$ for 20 minutes in microwave.

Primary antibodies were incubated $\mathrm{ON}$ at RT in a humidified chamber. The slides were analyzed using an optic microscope (BX40, Olympus Optical Corporation, Tokyo, Japan) and imaged using a coupled digital camera (390CU 3.2 Mega Pixel CCD Camera, Micrometrics, Spain). 
To calculate morphometric descriptors cell outlines were used. The analysis was performed on images of cells stained with crystal violet taken with an inverted microscope. Two hundred cells were analyzed for each cell line. The quantification of each shape descriptor was performed using ImageJ (NIH, Bethesda, $\mathrm{MA})$. Elongation was defined as ratio of length (maximum diameter) and the width of the cell, and Roundness was calculated as $(4 \mathrm{x}$ area $) /(\pi \times \text { perimeter })^{2}$.

\section{Transwell migration assays.}

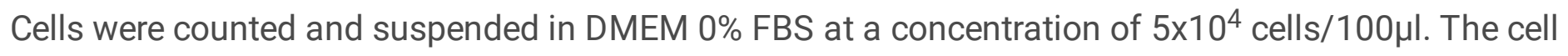
suspension was seeded on the upper chamber of a 24-well $0.8 \mu \mathrm{m}$ pore size Cell Culture Insert (BD). The bottom side of each insert was previously filled with $700 \mu$ l of DMEM $10 \%$ FBS. After 16 or $20 \mathrm{~h}$ (depending on the experiment), inserts were removed, washed, and cells that had migrated to the bottom side of the inserts were fixed with $4 \%$ PFA and stained with $0.1 \%$ crystal violet in $10 \%$ ethanol. Pictures were taken on Olympus Q-Colour.5 and the number of cells per field was counted.

\section{Bioinformatic analysis}

ROR2 was tested for association with prognostic signatures (LMC classes) [26] derived from expression data from the Leeds Melanoma Cohort (LMC) [49]. STATA 14 (StataCorp, Texas, USA) was used to conduct the Mann-Whitney test for group comparisons. We used three additional gene expression datasets (GSE65904, GSE46517, and E-TABM-4) that are available from the Gene Expression Omnibus (GEO) and the ArrayExpress databases. Dataset GSE65904 ( $n=214)$ was used to assess the link between prognosis and ROR2 expression in lymph node metastasis (LNM). To avoid the artificial effects of assigning patients with similar expression levels to different groups, a Z-score cut-off of 0.2 and -0.2 was used to separate patients with high and low expression, respectively. Datasets GSE46517 ( $\mathrm{n}=121)$ and ETABM-4, together with The Cancer Genome Atlas (SKCM-TCGA) dataset were used to compare ROR2 expression in primary and metastatic melanoma. Identification of Differentially Expressed Genes (DEGs) on dataset GSE65904 was performed using GEO2R. Three hundred and twenty one DEGs with an adjusted $p$-value $<0.05$ and a logFC $>1$ were selected for further analysis. Gene enrichment was performed using ShinyGO [50].

\section{Statistics.}

All experiments were performed at least three times. A mean and standard error was derived from all repeated experiments. Student's t-test and ANOVA were performed to compare groups. Values of $p<0.05$ were considered statistically significant. Statistical analyses were conducted using software from GraphPad Prism. The number of independent experiments and specific statistical analyses used in each experiment are indicated in the figure legends.

\section{Abbreviations}


EMT, epithelial-to-mesenchymal transition; FFPE, formalin-fixed paraffin embedded, MSS, melanomaspecific survival; LMC, Leeds melanoma cohort, GEO, Gene Expression Omnibus; DEG, Differentially Expressed Genes; PLX, PLX-4032, LNM, lymph node metastasis; dMFS, distant metastasis-free survival.

\section{Declarations}

\section{Ethics approval}

Animal studies were approved by the Institutional Committee for the care and use of experimental animals.

\section{Competing interests}

The authors declare that they have no competing interests

\section{Availability of data and materials}

Data sharing is not applicable to this article as no datasets were generated during the current study.

\section{Funding}

This research was supported by grants BID-PICT-2015-0513 and BID-PICT-2011-1605 from the Agencia Nacional de Promoción Científica y Tecnológica (ANPCyT) and grants from Fundación Científica Felipe Fiorellino. Consejo Nacional de Investigaciones Científicas y Técnicas (CONICET) provided fellowships to MVC, GB, MBV, and MJQ. The Leeds Melanoma Cohort was built using grants CR UK C588/A19167, C8216/A6129 and C588/A10721 and NIH CA83115.

\section{Author contributions}

MVC performed most of the experiments and was responsible for analysis and visualization of the data. GAB contributed to the experiments and the methodology. MBV helped with the methodology. JN and JNB conducted the analysis of ROR2 expression in the LMC. El contributed with the analysis of IHC data. MJQ contributed to the experiments. PLB was responsible for conceptualization, funding acquisition and project administration. He was also responsible for writing and editing the manuscript. All the authors provided feedback on the report.

\section{Acknowledgements}

We thank Miss Alejandra Fisz for administrative assistance and Engr. Alberto Varela and his crew for technical assistance. We thank Sara Correa and Diego Vazquez for expert assistance with histotechniques.

\section{References}


1. R. L. Siegel, K. D. Miller, and A. Jemal, CA A Cancer J Clin 70, 7 (2020).

2. S. Ugurel, J. Röhmel, P. A. Ascierto, K. T. Flaherty, J. J. Grob, A. Hauschild, J. Larkin, G. V. Long, P. Lorigan, G. A. McArthur, A. Ribas, C. Robert, D. Schadendorf, and C. Garbe, European Journal of Cancer 83, 247 (2017).

3. R. Kalluri and R. A. Weinberg, J Clin Invest 119, 1420 (2009).

4. U. D. Kahlert, J. V. Joseph, and F. A. E. Kruyt, Mol Oncol 11, 860 (2017).

5. D. Pedri, P. Karras, E. Landeloos, J. Marine, and F. Rambow, FEBS J febs.16021 (2021).

6. E. Kang, J. Seo, H. Yoon, and S. Cho, Int J Mol Sci 22, 3591 (2021).

7. J. Caramel, E. Papadogeorgakis, L. Hill, G. J. Browne, G. Richard, A. Wierinckx, G. Saldanha, J. Osborne, P. Hutchinson, G. Tse, J. Lachuer, A. Puisieux, J. H. Pringle, S. Ansieau, and E. Tulchinsky, Cancer Cell 24, 466 (2013).

8. Z. Debebe and W. K. Rathmell, Pharmacology \& Therapeutics 150, 143 (2015).

9. C. E. Ford, S. S. Qian Ma, A. Quadir, and R. L. Ward, Int J Cancer 133, 779 (2013).

10. B. Sun, X. Ye, L. Lin, M. Shen, and T. Jiang, Int J Clin Exp Pathol 8, 856 (2015).

11. C. Yang, S. Ji, Y. Li, L. Fu, T. Jiang, and F. Meng, Oncol Res 25, 195 (2017).

12. T. M. Wright, A. R. Brannon, J. D. Gordan, A. J. Mikels, C. Mitchell, S. Chen, I. Espinosa, M. van de Rijn, R. Pruthi, E. Wallen, L. Edwards, R. Nusse, and W. K. Rathmell, Oncogene 28, 2513 (2009).

13. E. Lara, V. Calvanese, C. Huidobro, A. F. Fernández, Á. Moncada-Pazos, Á. J. Obaya, O. Aguilera, J. González-Sancho, L. Sánchez, A. Astudillo, A. Muñoz, C. López-Otín, M. Esteller, and M. F. Fraga, Mol Cancer 9, 170 (2010).

14. M. P. O'Connell, J. L. Fiori, M. Xu, A. D. Carter, B. P. Frank, T. C. Camilli, A. D. French, S. K. Dissanayake, F. E. Indig, M. Bernier, D. D. Taub, S. M. Hewitt, and A. T. Weeraratna, Oncogene 29, 34 (2010).

15. C. Henry, A. Quadir, N. J. Hawkins, E. Jary, E. Llamosas, D. Kumar, B. Daniels, R. L. Ward, and C. E. Ford, J. Cancer Res. Clin. Oncol. 141, 243 (2015).

16. C. E. Henry, E. Llamosas, B. Daniels, A. Coopes, K. Tang, and C. E. Ford, Gynecol. Oncol. 148, 576 (2018).

17. S. S. Q. Ma, S. Srivastava, E. Llamosas, N. J. Hawkins, L. B. Hesson, R. L. Ward, and C. E. Ford, BMC Cancer 16, 508 (2016).

18. L. Yan, Q. Du, J. Yao, and R. Liu, Exp Ther Med 12, 4128 (2016).

19. S. E. Lee, S. D. Lim, S. Y. Kang, S. B. Suh, and Y.-L. Suh, Brain Pathol. 23, 445 (2013).

20. M. Nishita, S. K. Yoo, A. Nomachi, S. Kani, N. Sougawa, Y. Ohta, S. Takada, A. Kikuchi, and Y. Minami, J. Cell Biol. 175, 555 (2006).

21. S.-S. Lai, B. Xue, Y. Yang, L. Zhao, C.-S. Chu, J.-Y. Hao, and C.-J. Wen, Cancer Genet 205, 552 (2012).

22. M. P. O'Connell, K. Marchbank, M. R. Webster, A. A. Valiga, A. Kaur, A. Vultur, L. Li, M. Herlyn, J. Villanueva, Q. Liu, X. Yin, S. Widura, J. Nelson, N. Ruiz, T. C. Camilli, F. E. Indig, K. T. Flaherty, J. A. 
Wargo, D. T. Frederick, Z. A. Cooper, S. Nair, R. K. Amaravadi, L. M. Schuchter, G. C. Karakousis, W. Xu, X. Xu, and A. T. Weeraratna, Cancer Discov 3, 1378 (2013).

23. S. R. Alonso, L. Tracey, P. Ortiz, B. Pérez-Gómez, J. Palacios, M. Pollán, J. Linares, S. Serrano, A. I. Sáez-Castillo, L. Sánchez, R. Pajares, A. Sánchez-Aguilera, M. J. Artiga, M. A. Piris, and J. L. Rodríguez-Peralto, Cancer Res 67, 3450 (2007).

24. S. M. Lyons, E. Alizadeh, J. Mannheimer, K. Schuamberg, J. Castle, B. Schroder, P. Turk, D. Thamm, and A. Prasad, Biol Open 5, 289 (2016).

25. Y. Tang, S. Durand, S. Dalle, and J. Caramel, Cancers 12, 2154 (2020).

26. R. Thakur, J. P. Laye, M. Lauss, J. M. S. Diaz, S. J. O’Shea, J. Poźniak, A. Filia, M. Harland, J. Gascoyne, J. A. Randerson-Moor, M. Chan, T. Mell, G. Jönsson, D. T. Bishop, J. Newton-Bishop, J. H. Barrett, and J. Nsengimana, Clin Cancer Res 25, 7424 (2019).

27. J. Xu, J. Shi, W. Tang, P. Jiang, M. Guo, B. Zhang, and G. Ma, J Cell Biochem jcb.29666 (2020).

28. K. D. Marshall, M. A. Edwards, M. Krenz, J. W. Davis, and C. P. Baines, Am J Physiol Cell Physiol 306, C639 (2014).

29. S. Gobeil, C. C. Boucher, D. Nadeau, and G. G. Poirier, Cell Death Differ 8, 588 (2001).

30. L. Li, J. Ying, X. Tong, L. Zhong, X. Su, T. Xiang, X. Shu, R. Rong, L. Xiong, H. Li, A. T. C. Chan, R. F. Ambinder, Y. Guo, and Q. Tao, Cell. Mol. Life Sci. 71, 2179 (2014).

31. X. Wu, T. Yan, L. Hao, and Y. Zhu, Cancer Manag Res 11, 2803 (2019).

32. T. Sakamoto, S. Kawano, R. Matsubara, Y. Goto, T. Jinno, Y. Maruse, N. Kaneko, Y. Hashiguchi, T. Hattori, S. Tanaka, R. Kitamura, T. Kiyoshima, and S. Nakamura, Oral Oncol. 69, 15 (2017).

33. D. Ren, Y. Minami, and M. Nishita, Genes to Cells 16, 304 (2011).

34. T. Bland, J. Wang, L. Yin, T. Pu, J. Li, J. Gao, T.-P. Lin, A. C. Gao, and B. J. Wu, IScience 24, 101970 (2021).

35. X. Kong, T. Kuilman, A. Shahrabi, J. Boshuizen, K. Kemper, J.-Y. Song, H. W. M. Niessen, E. A. Rozeman, M. H. Geukes Foppen, C. U. Blank, and D. S. Peeper, Nature 550, 270 (2017).

36. A. Hong, G. Moriceau, L. Sun, S. Lomeli, M. Piva, R. Damoiseaux, S. L. Holmen, N. E. Sharpless, W. Hugo, and R. S. Lo, Cancer Discov 8, 74 (2018).

37. G. P. Leung, T. Feng, F. D. Sigoillot, F. C. Geyer, M. D. Shirley, D. A. Ruddy, D. P. Rakiec, A. K. Freeman, J. A. Engelman, M. Jaskelioff, and D. D. Stuart, Mol Cancer Res 17, 199 (2019).

38. B. N. Smith, L. J. Burton, V. Henderson, D. D. Randle, D. J. Morton, B. A. Smith, L. Taliaferro-Smith, P. Nagappan, C. Yates, M. Zayzafoon, L. W. K. Chung, and V. A. Odero-Marah, PLoS ONE 9, e104987 (2014).

39. C. H. Kim, H. M. Jeon, S. Y. Lee, M. K. Ju, J. Y. Moon, H. G. Park, M.-A. Yoo, B. T. Choi, J. I. Yook, S.-C. Lim, S. I. Han, and H. S. Kang, PLoS ONE 6, e18000 (2011).

40. S. Y. Lee, M. K. Ju, H. M. Jeon, E. K. Jeong, Y. J. Lee, C. H. Kim, H. G. Park, S. I. Han, and H. S. Kang, Oxidative Medicine and Cellular Longevity 2018, 1 (2018).

41. Z.-G. Liu and D. Jiao, Cell Stress 4, 1 (2019). 
42. T. M. Wright and W. K. Rathmell, Journal of Biological Chemistry 285, 12916 (2010).

43. L. S. Campos, Y. I. Rodriguez, A. M. Leopoldino, N. C. Hait, P. Lopez Bergami, M. G. Castro, E. S. Sanchez, M. Maceyka, S. Spiegel, and S. E. Alvarez, Mol. Cell. Biol. 36, 320 (2016).

44. M. E. Picco, M. V. Castro, M. J. Quezada, G. Barbero, M. B. Villanueva, N. B. Fernández, H. Kim, and P. Lopez-Bergami, Cell Biosci 9, 3 (2019).

45. S. DeMorrow, H. Francis, E. Gaudio, J. Venter, A. Franchitto, S. Kopriva, P. Onori, R. Mancinelli, G. Frampton, M. Coufal, B. Mitchell, B. Vaculin, and G. Alpini, Am J Physiol Gastrointest Liver Physiol 295, G1150 (2008).

46. N. B. Fernández, D. Lorenzo, M. E. Picco, G. Barbero, L. S. Dergan-Dylon, M. P. Marks, H. GarcíaRivello, L. Gimenez, V. Labovsky, L. Grumolato, and P. Lopez-Bergami, Mol. Carcinog. 55, 1772 (2016).

47. G. Barbero, M. V. Castro, M. B. Villanueva, M. J. Quezada, N. B. Fernández, S. DeMorrow, and P. LopezBergami, Cells 8, 1060 (2019).

48. M. J. Quezada, M. E. Picco, M. B. Villanueva, M. V. Castro, G. Barbero, N. B. Fernández, E. Illescas, and P. Lopez-Bergami, Cancers 13, 78 (2020).

49. J. Nsengimana, J. Laye, A. Filia, S. O’Shea, S. Muralidhar, J. Poźniak, A. Droop, M. Chan, C. Walker, L. Parkinson, J. Gascoyne, T. Mell, M. Polso, R. Jewell, J. Randerson-Moor, G. P. Cook, D. T. Bishop, and J. Newton-Bishop, J Clin Invest 128, 2048 (2018).

50. S. X. Ge, D. Jung, and R. Yao, Bioinformatics 36, 2628 (2020).

\section{Supplementary}

Supplementary tables are not available with this version

\section{Figures}


Figure 1
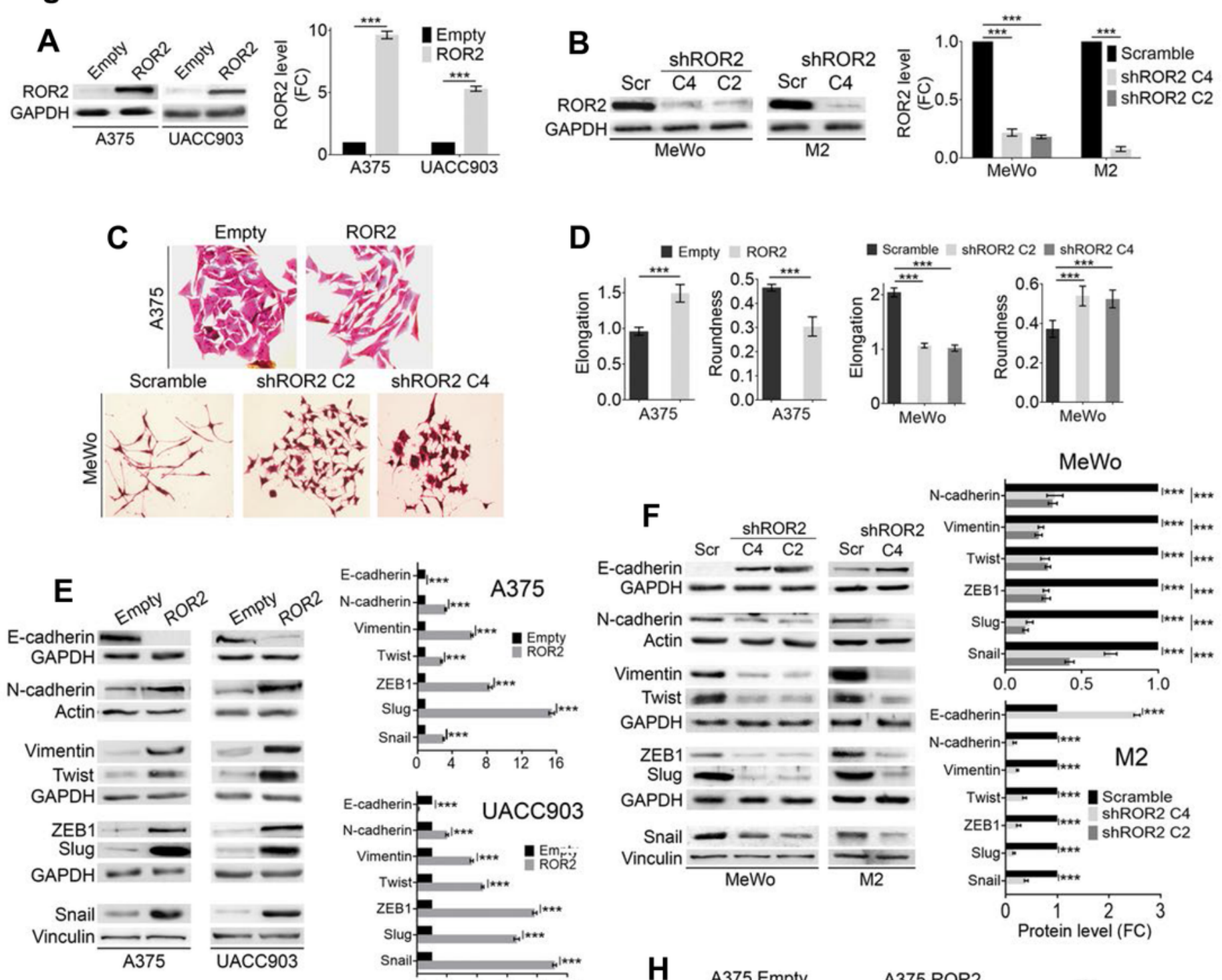

E-cadherin-1

A375
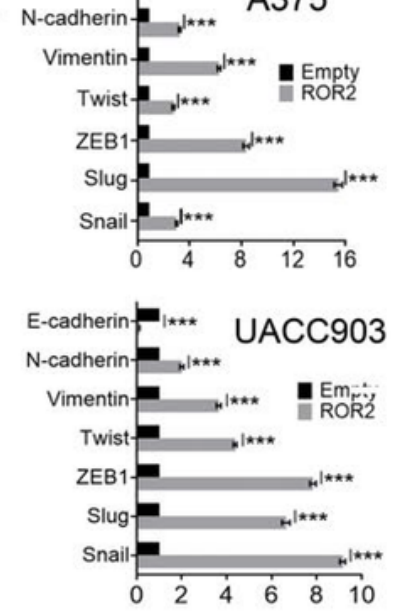

H
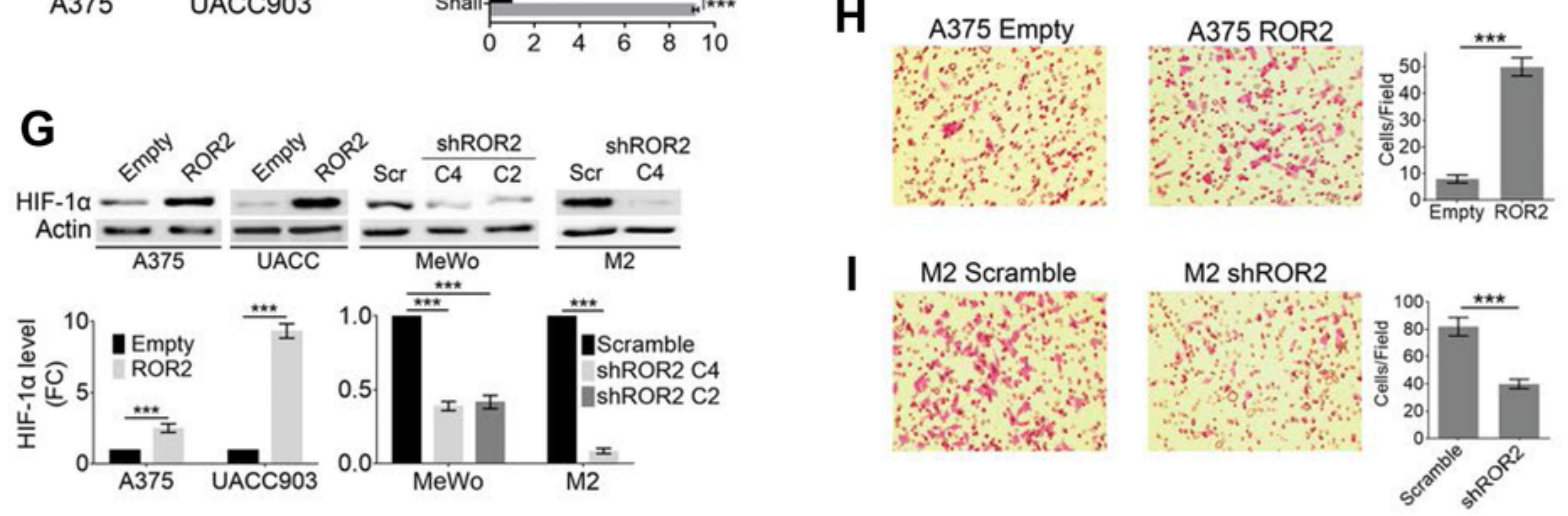

\section{Figure 1}

ROR2 promotes EMT and migration of melanoma cells. (A) Overexpression of ROR2 in A375 and UACC903 cell lines. ROR2 expression was assessed by western blot in cells stably transduced with either control (Empty) or a ROR2-expressing plasmid. The graphs show the mean \pm S.D. of ROR2 levels normalized to GAPDH levels and expressed as the fold change $(F C)$ relative to the corresponding control cell line (Empty). (B) Silencing of ROR2 in M2 and MeWo cells. ROR2 expression was assessed by 
western blot in cells stably transduced with either control (scramble) or two shRNA for ROR2 (C4 and C2). Data were analyzed as described in A. (C, D) ROR2 induces morphological changes in melanoma cells. (C) Phase contrast images. (D) Quantification of elongation and roundness $(n=200)$. (E) ROR2 promotes the expression of EMT markers. Evaluation of the expression of the indicated EMT markers in cells described in A. The graphs show the mean \pm S.D. of each protein's levels normalized to the corresponding loading control and expressed as the fold change $(\mathrm{FC})$ relative to the corresponding control cell line (Empty). (F) ROR2 silencing inhibits expression of mesenchymal markers. Evaluation of the expression of the indicated EMT markers in cells described in B. The graphs show the mean \pm S.D. of each protein's levels normalized to the corresponding loading control and expressed as the fold change (FC) relative to the corresponding control cell line (scramble). (G) ROR2 promotes the expression of HIF-1a. Analysis of HIF-1a expression in cell lines described in A and B. The graphs show the mean \pm S.D. of HIF-1a levels normalized to the corresponding loading control and expressed as the fold change $(\mathrm{FC})$ relative to the corresponding control cell line (Empty or scramble). (H) ROR2 increases melanoma cell migration. Cells were allowed to migrate for $20 \mathrm{~h}$. Representative images of cells that migrated through the pores of the transwell chamber are shown. Graphs show the mean \pm S.D. of the number of cells per field. (I) ROR2 silencing inhibits melanoma cell migration. Cells were allowed to migrate for $16 \mathrm{~h}$. Data were analyzed as in $\mathrm{H}$. Statistical significance was tested by a one-tailed Student's T-Test or ANOVA as appropriate. $\mathrm{n}=3$ unless otherwise indicated. $* \star *: p<0.0001$.

\section{Figure 2}

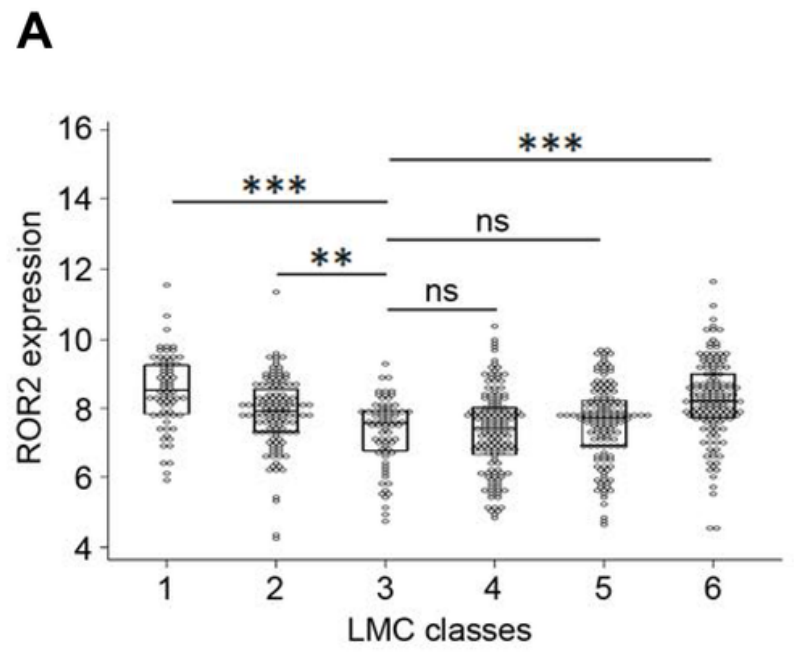

B

\begin{tabular}{|c|c|}
\hline Functional Category & $\begin{array}{l}\text { Enrichment } \\
\quad \text { FDR }\end{array}$ \\
\hline MSigDB:MODULE 47 & $5.16 \mathrm{E}-108$ \\
\hline MSigDB:SCHUETZ BREAST CANCER DUCTAL INVASIVE UP & 4.36E-86 \\
\hline MSigDB:BOQUEST STEM CELL UP & $2.15 \mathrm{E}-75$ \\
\hline MSigDB:HALLMARK EPITHELIAL MESENCHYMAL TRANSITION & 8.32E-71 \\
\hline MSigDB:NABA CORE MATRISOME & $2.76 \mathrm{E}-70$ \\
\hline $\begin{array}{l}\text { Extracellular matrix } \\
\text { MSigDB:ANASTASSIOU MULTICANCER INVASIVENESS }\end{array}$ & 1.07E-68 \\
\hline SIGNATURE & $1.60 \mathrm{E}-68$ \\
\hline Collagen-containing extracellular matrix & 1.37E-61 \\
\hline Extracellular matrix structural constituent & $9.78 \mathrm{E}-59$ \\
\hline Extracellular matrix organization & $9.78 E-59$ \\
\hline MSigDB:NABA MATRISOME & $2.10 \mathrm{E}-58$ \\
\hline Extracellular structure organization & $8.20 \mathrm{E}-58$ \\
\hline MSigDB:MODULE 1 & 4.89E-56 \\
\hline
\end{tabular}

Figure 2

ROR2 associates with EMT signatures in melanoma patients. (A) ROR2 mRNA expression is strongest in LMC class 6 which has activation of EMT and poor survival. LMC class 3 with the worst survival was used as a baseline for comparison [26]. Mann-Whitney test for group comparisons was used. ns: not 
significant, $\star *: p<0.001, * \star *: p<0.0001$. (B) Biological processes enriched in DEGs upregulated in patients with high ROR2 levels from dataset GSE65904.

\section{Figure 3}

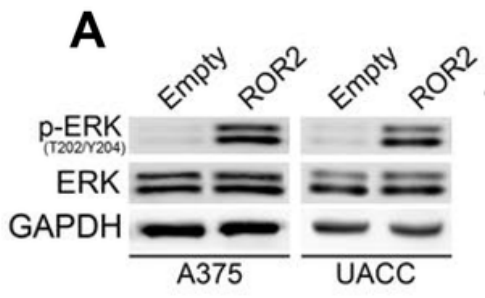

C

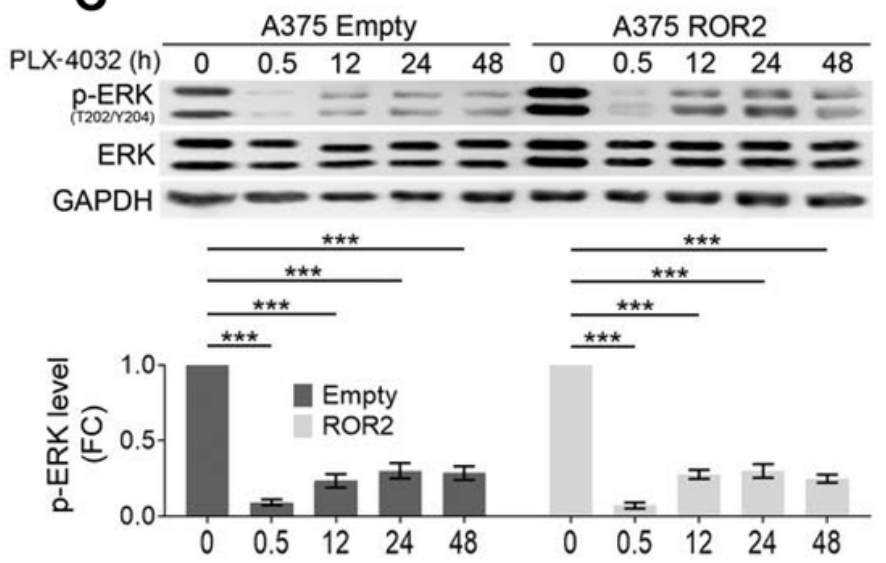

$\mathbf{E}$

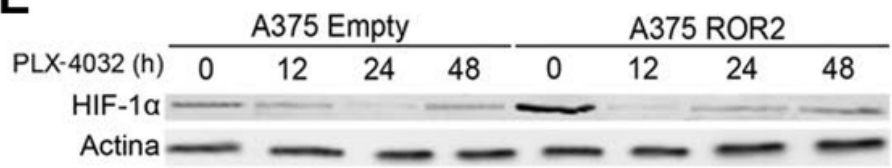

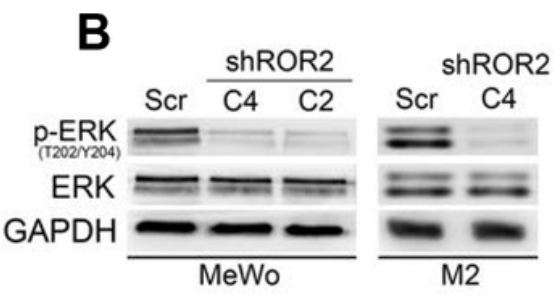
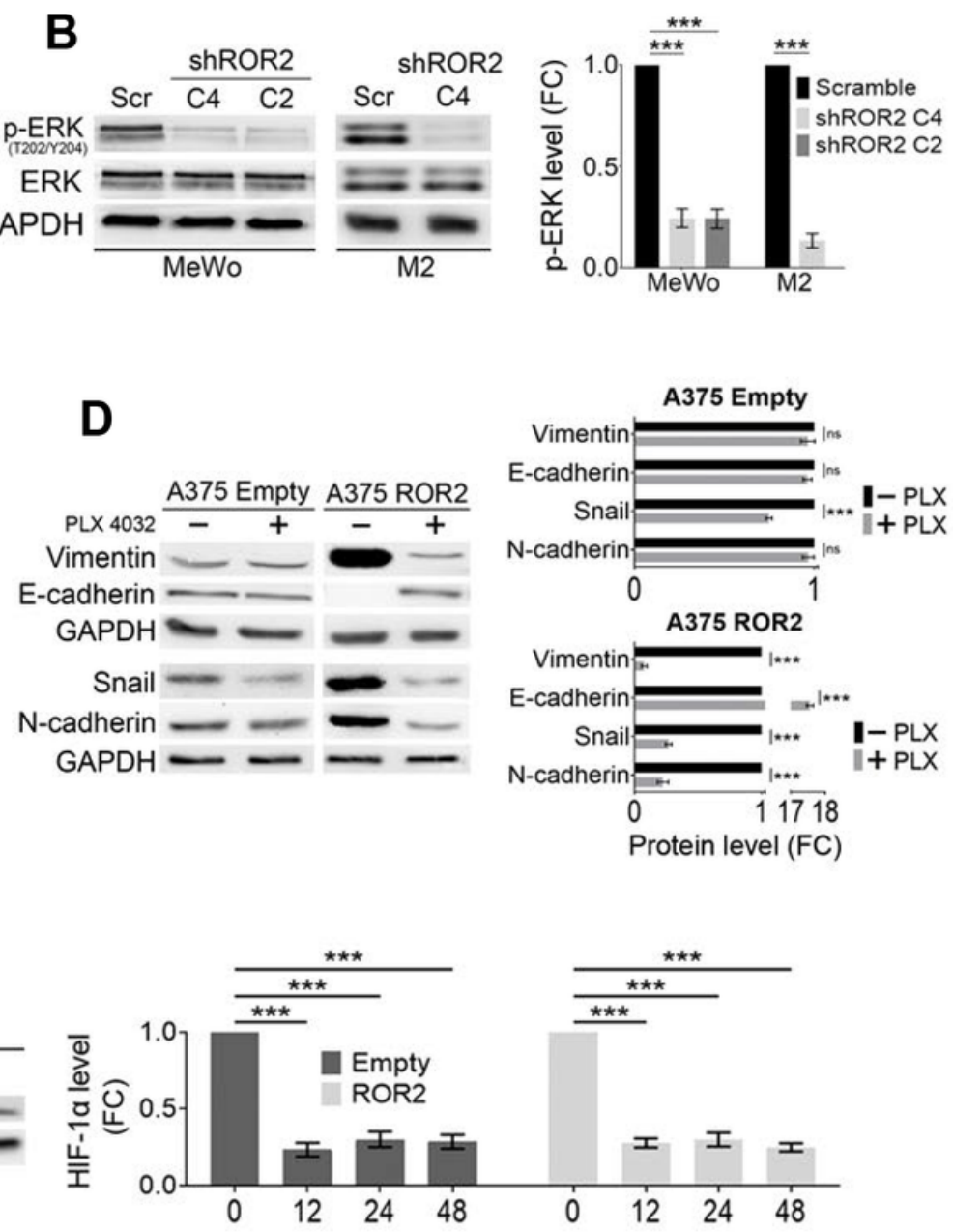

\section{Figure 3}

EMT induction by ROR2 is mediated by increased ERK phosphorylation. (A) ROR2 increases ERK phosphorylation. Western blot analysis of p-ERK levels in A375 and UACC903 cells lines expressing ROR2. The graphs show the mean \pm S.D. of $p$-ERK level normalized to total ERK levels and expressed as the fold change (FC) relative to the corresponding control cell line (Empty). (B) ROR2 silencing inhibits ERK phosphorylation. Western blot analysis of p-ERK levels in M2 and MeWo cells upon ROR2 silencing. The graphs show the mean \pm S.D. of $p$-ERK level normalized to total ERK levels and expressed as the fold change (FC) relative to the corresponding control cell line (scramble). (C) PLX similarly inhibits p-ERK in A375-Empty and A375-ROR2 cells. A375-Empty and A375-ROR2 cells were incubated with $10 \mu \mathrm{M}$ PLX for the indicated times and protein extracts were analyzed by western blot with the indicated antibodies. GAPDH was used as loading control. The graph shows the mean \pm S.D. of $p$-ERK levels in ROR2- 
expressing cells normalized to total ERK levels and expressed as the fold change (FC) relative to untreated cells. (D) PLX inhibits the increase in EMT markers induced by ROR2. The cells were treated with $10 \mu \mathrm{M}$ PLX for $12 \mathrm{~h}$. The graphs show the mean \pm S.D. of each protein's levels normalized to the corresponding loading control and expressed as the fold change $(\mathrm{FC})$ relative to untreated cells. (E) HIF1a expression is regulated by the MAPK/ERK pathway. PLX inhibits HIF-1a expression in both A375Empty and A375-ROR2 cells. The cells were treated with $10 \mu \mathrm{M}$ PLX for the indicated times. The graphs show the mean \pm S.D. of HIF-1 a levels normalized to the corresponding loading control and expressed as the fold change $(\mathrm{FC})$ relative to untreated cells. Statistical significance was tested by a one-tailed Student's T-Test or ANOVA as appropriate $(n=3)$. ***: $p<0.0001$, n.s.: no significant.

\section{Figure 4}

A

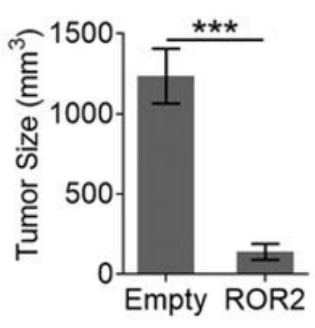

B
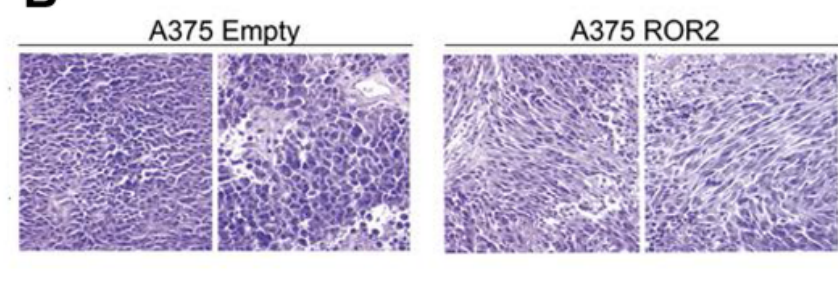

D

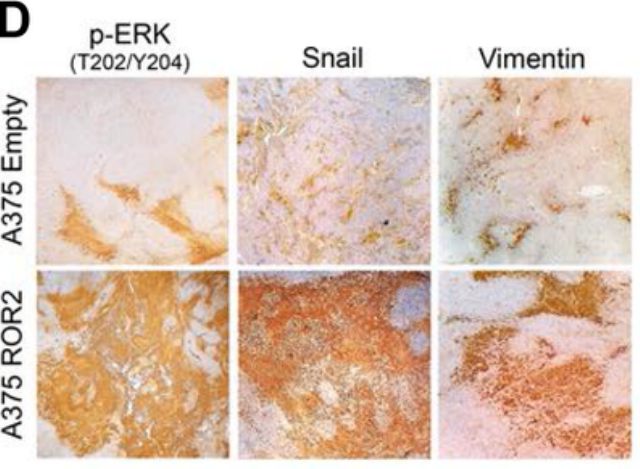

E

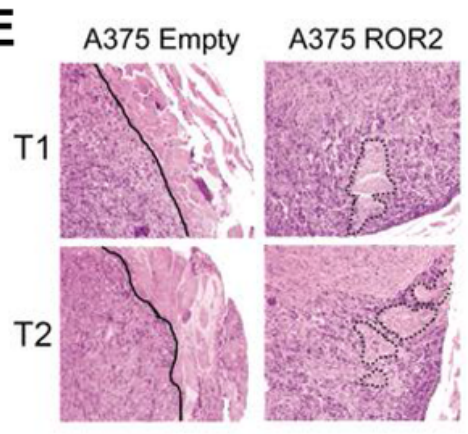

\section{Figure 4}

ROR2 induces EMT and invasion in vivo. (A) ROR2 expression inhibits tumor growth. A375-Empty and A375-ROR2 cells were xenotransplanted in nude mice (6 per group) and tumor volume was monitored twice weekly. The graph show the mean \pm S.D. of the tumor volume after six weeks. Statistical significance was tested by a one-tailed Student's T-Test, $\star \star \star: ~ p<0.0001$. (B) Tumor cells from mice injected with A375-ROR2 present an elongated shape. Histological analysis of representative H\&E-stained FFPE sections (200x magnification). (C) Tumors from mice injected with A375-ROR2 present increased levels of $p$-ERK and of EMT markers. Western blot analysis of the indicated proteins in tumors extracts (four mice per group). Actin and GAPDH were used as loading controls. (D) Tumors expressing ROR2 show massive expression of p-ERK, Snail and Vimentin. Representative images at 40x magnification of IHC 
staining of tumor sections. (E) Infiltration of fibromuscular tissue by melanoma cells in tumors from A375-ROR2 injected mice. Solid lines show the tumor-muscle interface in A375-Empty tumors. Tumor cells invading muscle tissue were not observed. In A375-ROR2 tumors, dotted lines indicate regions of muscle tissue surrounded by invading tumor cells. Representative images at $100 x$ magnification are shown.

\section{Figure 5}

A
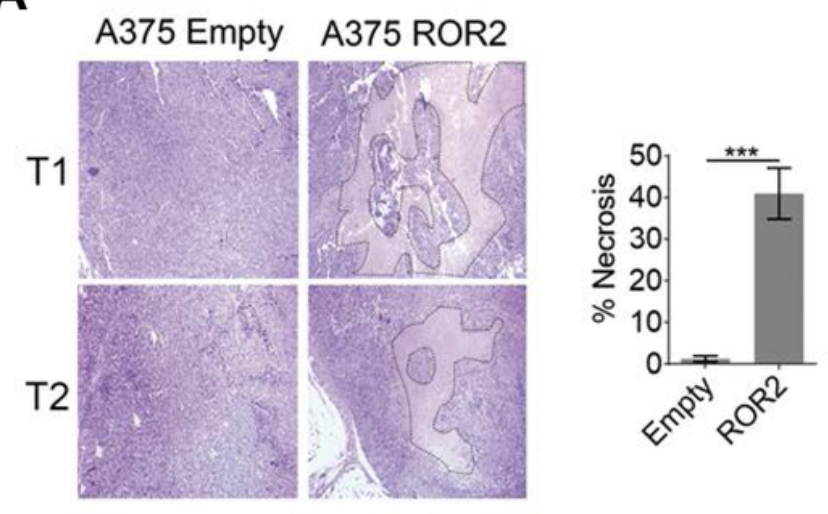

B
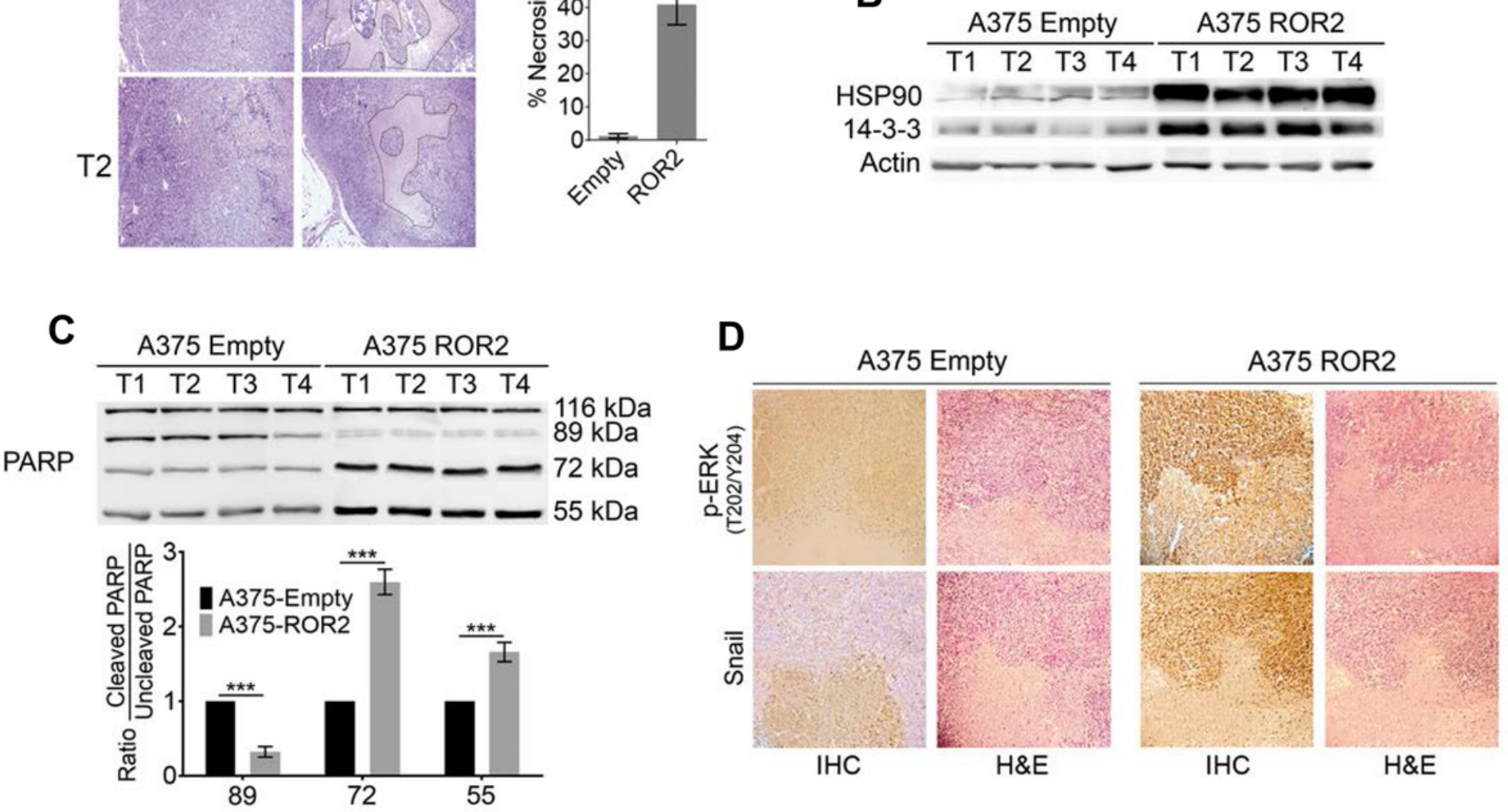

\section{Figure 5}

Tumors from mice injected with A375-ROR2 cells presents extensive necrosis. (A) Histological analysis of representative H\&E-stained FFPE sections from A375-ROR2 tumors show extensive areas of necrosis (dotted lines) at low magnification (40x). Graph shows the mean \pm S.D. of the relative area of necrosis. (B) Tumors from mice injected with A375-ROR2 display increased expression of HSP90 and 14-3-3. Western blot analysis of the indicated proteins in tumors extracts (four mice per group). Actin was used as loading control. (C) Tumors from mice injected with A375-ROR2 display increased necrotic cleavage of 
PARP. Western blot analysis of PARP expression in tumors extracts (four mice per group). Uncleaved PARP $(116 \mathrm{kDa})$ and three PARP cleaved forms $(89,72$, and $55 \mathrm{kDa})$ are shown. Cleavage of PARP that give rise to 72 and $55 \mathrm{kDa}$ forms is the result of necrotic cell death [29]. The graph shows the mean \pm S.D. of each cleaved PARP forms normalized to the amount of uncleaved PARP and expressed as the fold change relative to control (A375-Empty) tumors. Statistical significance was tested by a one-tailed Student's T-Test. ***: $p<0.0001$. (D) A375-ROR2 tumors present strong nuclear $p$-ERK and nuclear Snail staining adjacent to necrotic areas. Representative images at $40 x$ magnification of both $\mathrm{IHC}$ and $\mathrm{H} \& \mathrm{E}$ staining of tumor sections.

\section{Figure 6}
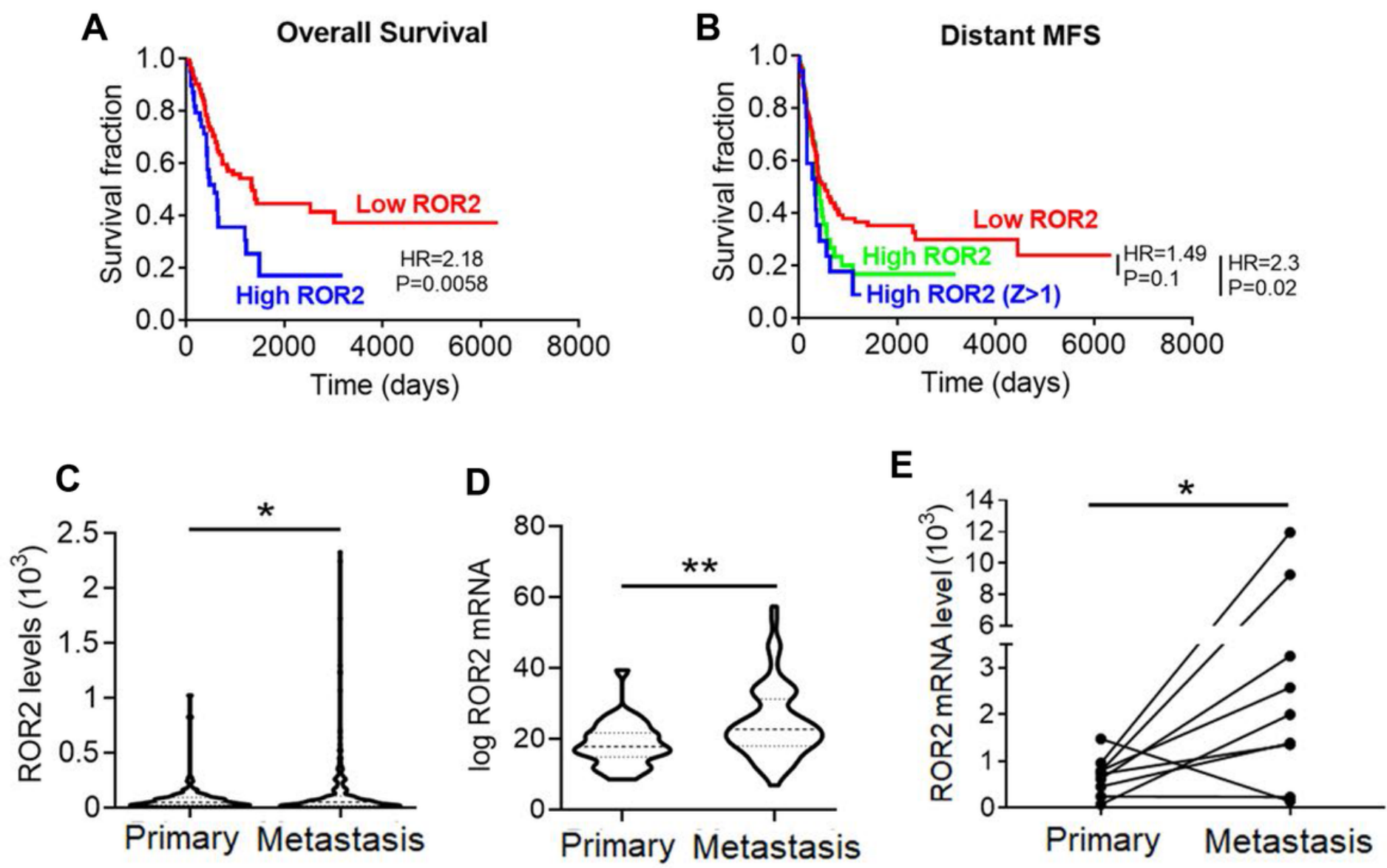

Figure 6

ROR2 expression in lymph node metastasis correlates with shorter survival. (A) Melanoma patients presenting LNM with high ROR2 levels have shorter overall survival. Overall survival curves of melanoma patients with low (Z-score $<-0.2, n=105)$ and high (Z-score $>0.2, n=40)$ ROR2 levels. (B) Distant metastasis-free survival curves of melanoma patients with low (Z-score <-0.2, $n=100)$, high (Z-score > $0.2, n=37$ ) and very high ( $Z$-score $>1, n=17)$ ROR2 levels. Data for $(A)$ and $(B)$ are from the GSE65904 dataset. Statistical significance was determined by the Log-rank Test. Hazard Ratio (HR) and P are 
shown. (C) Analysis of ROR2 mRNA levels in melanoma samples. Violin plot showing ROR2 mRNA expression in 103 primary melanoma and 361 metastatic melanomas from the TCGA-SKCM dataset. Statistical significance was determined by an unpaired T-Test with Welch's correction for unequal variance. $*: p<0.01$. (D) ROR2 expression is higher in metastasis than in primary tumors. Violin plots of ROR2 mRNA levels in samples ( $n=31$ and 73 , for primary and metastatic tumors, respectively) from the GSE46517 dataset. Statistical significance was tested by a one-tailed Student's T-Test. **: $p<0.001$. (E) ROR2 expression is higher in metastasis than in matched primary tumors. ROR2 mRNA levels from nine pairs of matched primary and metastatic samples from the dataset E-TABM-4 are connected with a line. Statistical significance was determined by a paired, one-tailed Student's T-Test using log transformed values. *: $p<0.01$. The plot shows untransformed data for a clearer visualization of the data points.

\section{Supplementary Files}

This is a list of supplementary files associated with this preprint. Click to download.

- SuppFigures.pdf 